\title{
Research, Development and Evaluation Capabilities for Betavoltaic Power Sources
}

\section{Dr. Thomas E Adams, NSWC Crane / Purdue University}

B.S. Electrical Engineering, University of Akron, 1987 M.S. Systems Engineering, Naval Postgraduate School, 2007 M.S. Nuclear Engineering, Purdue University, 2011 Ph.D. Nuclear Engineering, Purdue University, 2014

Employed by Naval Surface Warfare Center, Crane Division, on a PhD fellowship at Purdue University. Over 25 years experience in development and evaluation of power sources. Currently conducting R\&D on radioactive sources for power generation. Tom Adams has a strong background in battery power from his experience at NSWC Crane for 20 years. The experience includes testing and evaluation on batteries used in aircraft, missiles, hand-held devices and submarines, development of new battery designs and processes and design of equipment to measure the state of health of a battery. Since 2004, he has provided technical guidance on power sources for the Anti-Tamper/Software Protection Initiative (AT-SPI) by developing a power alternative guide for the design, and the validation and verification community. He is a member of the Inter-Agency Power Group (IAPG), American Society of Naval Engineers, and American Nuclear Society (ANS), American Society of Mechanical Engineers (ASME) and American Society of Engineering Education. He is currently working towards a Ph.D. in nuclear engineering in the area of direct conversion of radiation to electricity with the focus on semiconductor and emission efficiency.

\section{Prof. Shripad T Revankar, Purdue University, West Lafayette}

Dr. Revankar is a Professor of Nuclear Engineering at Purdue University. His main focus is on Energy -science and technology with sustained and distinguished achievements over the past three decades. His research intrests are on fuel cell, compact power sources including beta voltaic cells, multiphase flow and heat transfer, reactor thermalhydraulics, reactor safety, and nuclear hydrogen production. 


\section{Research, Development and Evaluation Capabilities for Betavoltaic Power Sources}

\section{Introduction}

Radiation interaction with materials can have beneficial uses, such as in betavoltaic cells, a type of radioisotope power source that utilize energy of beta radiation converted into electricity [1]. The specific development of betavoltaic devices has arisen out of the need for reliable, longlived, high energy density power sources for operating electrical systems in hostile and inaccessible environments, such as $-40^{\circ} \mathrm{C}$ and $80^{\circ} \mathrm{C}$. It is well established that conventional electrochemical batteries, despite their widespread use in electronic devices, have limited longevity and a strong tendency to degrade under extreme environmental conditions [2]. For situations where battery replacement is inconvenient or impossible, such as in remote sensing applications in space or aquatic environments, and where low-power generation can be utilized, the diminutive energy generated from a betavoltaic is suitable as an alternative to electrochemical battery technologies.

The Ragone plot of specific power (W/kg) versus specific energy (W-hr/kg) in Figure 1 illustrates where betavoltaic power fits in with other energy storage devices. In the upper left part of the plot, super capacitors dominate by delivering large amounts of power quickly. The bottom right represents devices that deliver low power for long periods, such as betavoltaics. Radioisotope thermoelectric generators (RTG) exhibit moderate power and operate for long periods as evident by the Voyager space probe, which is powered by several kilograms of plutonium-238. Lithium batteries provide high power, but typically operate for less than 5 years.

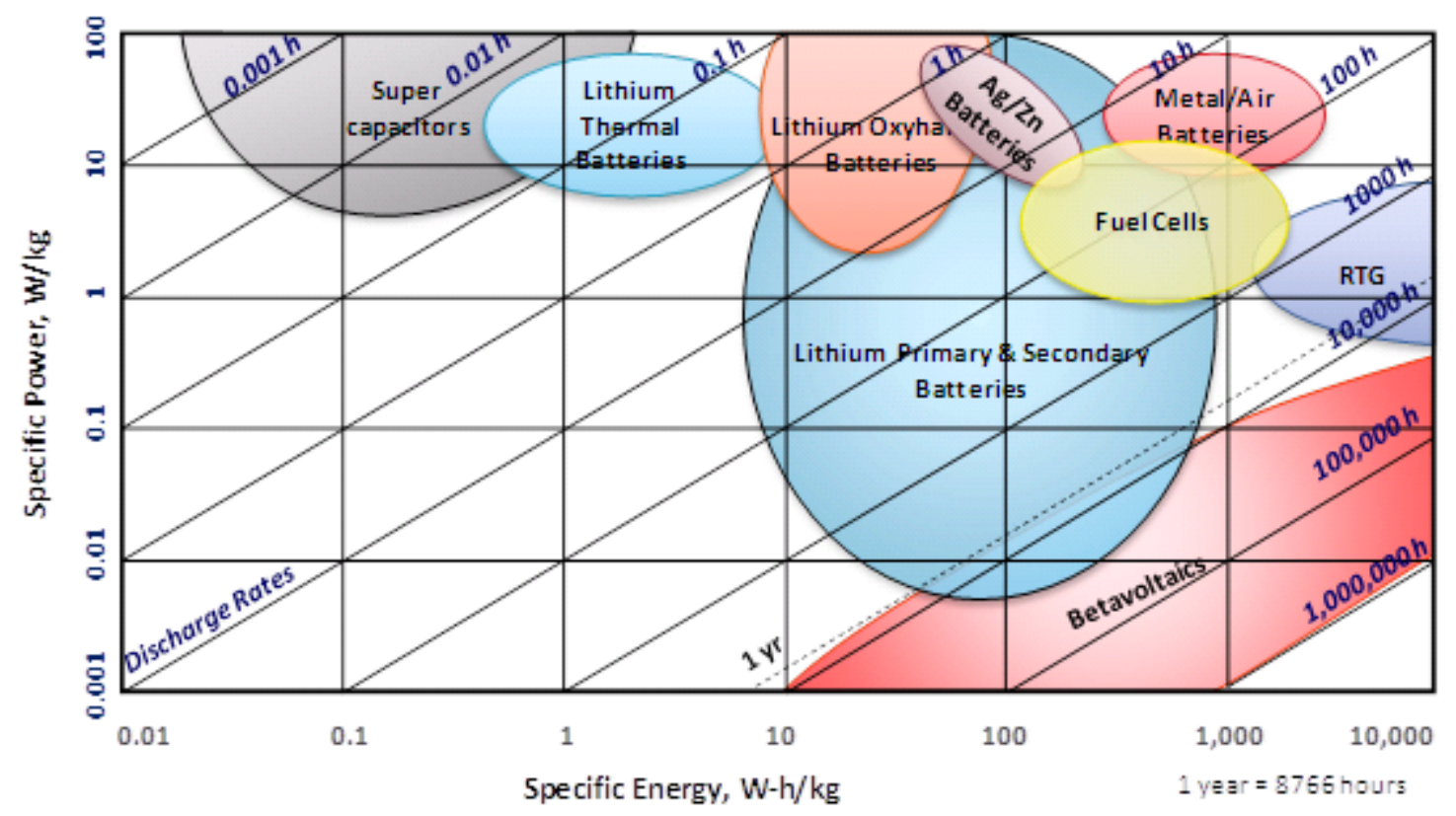

Figure 1. Ragone plot of energy storing devices: electrochemical batteries, capacitors, radioisotope thermoelectric generators (RTG) and betavoltaics 


\section{Background}

Radioisotopes are safely encountered in many applications shown in Figure 2. Smoke detectors rely on americium-241 $\left({ }^{241} \mathrm{Am}\right)$ for sensing smoke. Hospitals use $\mathrm{x}$-ray machines, chemotherapy, and tracers daily in many of the protocols. Tritium $\left({ }^{3} \mathrm{H}\right)$ is currently used to provide illumination, such as in exit signs, gun sights, and wristwatches. Promethium-147 $\left({ }^{147} \mathrm{Pm}\right)$ has been used to illuminate gauges for aircraft. Bananas naturally contain trace amounts of potassium- $40\left({ }^{40} \mathrm{~K}\right)$. Studies have also shown that an office building receives a larger dose of radiation from the Sun and space than inside a nuclear power plant. The stringent requirements of the Nuclear Regulatory Commission (NRC) often delay development until a general license or a sealed source and device permit is granted.

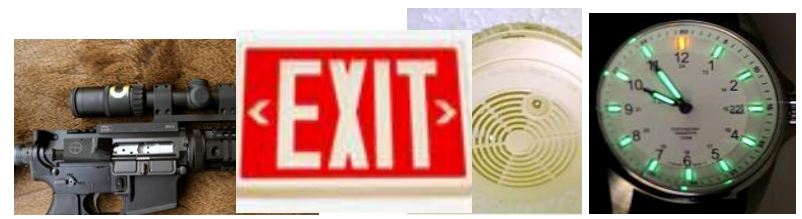

Figure 2. Examples of devices that use radioisotopes: gun sights $\left({ }^{3} \mathrm{H}\right)$, exit signs $\left({ }^{3} \mathrm{H}\right)$, smoke detectors $\left({ }^{241} \mathrm{Am}\right)$ and wrist watches $\left({ }^{3} \mathrm{H}\right)$

\subsection{Betavoltaic electrical conversion}

A direct conversion betavoltaic is comprised of two components, the p-n junction, and the beta source. Electricity is produced similar to a photovoltaic using the kinetic energy of the beta particles. The basic concept of operation is shown in Figure 3. Beta particles enter the p-n junction and collide with atoms creating electron-hole pairs (EHPs) as they slow down. A $5 \mathrm{keV}$ particle creates over 1000 EHPs, and deposits some energy into the lattice. In the depletion region, holes are accelerated to the $\mathrm{p}$-side collector and the electrons are accelerated to the $\mathrm{n}$-side collector. EHPs created outside the depletion layer quickly recombine and provide a net current of zero. With a load connected, the electrons travel from the n-side, through the load and back to the p-side. Because EHPs exhibit relatively short lives, the voltage developed is a function of the semiconductor band-gap and energy of the particle or photon.

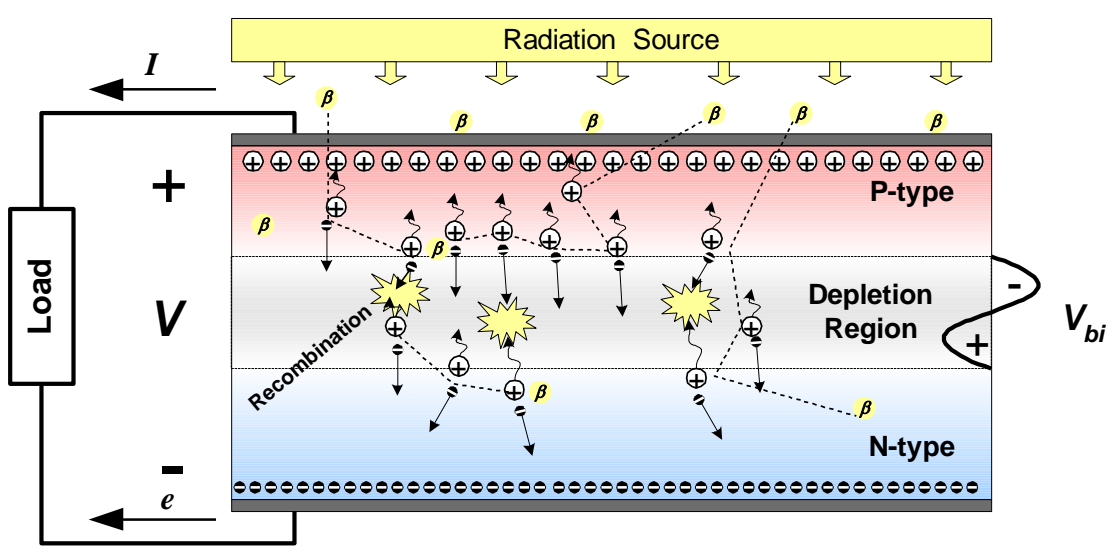

Figure 3. Voltaic Operation; conversion of radiation into electricity. 
Despite their operational similarities with photovoltaic devices, betavoltaic devices are usually strictly limited to low power applications [3]. This is directly related to the fact that the typical flux of beta particles emitted from a beta source is a minute fraction of the photon flux emitted by the sun. As a result, betavoltaic devices typically generate currents on the order of nano- to micro-amperes, which are several orders of magnitude smaller than currents generated by similarly sized photovoltaic devices $[4,5]$.

Betavoltaic electrical output characteristics are shown in Figure 4 and are measured by performing current-voltage (I-V) curves; a similar technique is performed on diodes and solar cells. Open-circuit voltage and short-circuit current are two important parameters for gauging performance. There are two basic modes of operation, constant current and constant voltage. In the voltage range from zero to $0.7 \mathrm{~V}$, the current does not appear to change and can be considered constant. Above the voltage of $0.85 \mathrm{~V}$, the current changes but the voltage changes little. In this range, the output voltage equals the difference between the open-circuit voltage and the resistance voltage; resistance of betavoltaics is in the $\mathrm{M} \Omega$ range. Most efficient operation occurs at peak power, which is at the knee of the I-V curve $(\sim 0.8 \mathrm{~V})$. The load lines indicate the impedance of the betavoltaic, which is maximum at open-circuit voltage and minimum at shortcircuit current. The mode of operation may be dependent on the matched impedance of the circuit. Many betavoltaics with similar operating performance can be stacked in parallel and series to provide higher currents and higher voltage, respectively. Temperature affects betavoltaics similar to the way it affects semiconductor electronics where the I-V curve shifts upward as temperature decreases and shifts downward as temperature increases.

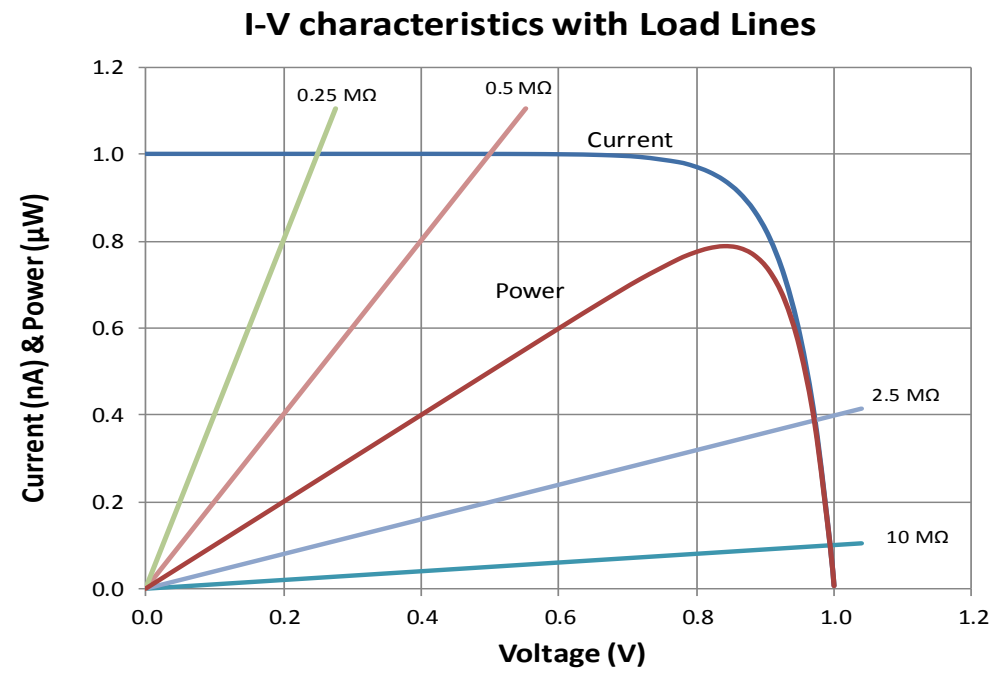

Figure 4. Betavoltaic I-V electrical characteristics

Hybrid designs using emerging lithium ion solid-state batteries with betavoltaics are being considered to provide latent and burst power. The betavoltaic is used to trickle charge the battery. 


\subsection{Status of betavoltaics}

The first betavoltaic battery was developed in 1953 at RCA by Rappaport. The device yielded an efficiency of only $0.2 \%$ and degraded rapidly due to radiation damage from the strontium-90 $\left({ }^{90} \mathrm{Sr}\right)$ radioactive source [6]. Several others continued research using ${ }^{147} \mathrm{Pm}$ but were only able to achieve efficiencies above $1 \%[2,7]$. The most promising effort in betavoltaics occurred in $c a$. 1974 through research led by Olsen at the Donald W. Douglas Laboratories [8, 9]. The Betacel battery, shown in Figure 5, exhibited $400 \mu \mathrm{W}$ and a $4 \%$ efficiency using ${ }^{147} \mathrm{Pm}$ and silicon p-n junctions. The Betacel was used in pacemakers that were implanted in over 285 patients, 60 inside the United States. German and U.S. medical institutions were seriously considering the Betacel for wider use. The United States Atomic Energy Commission (USAEC) had authorized the licensing of 50 Betacel pacemakers per month [10]. However, strides in lithium battery development entered onto the scene and were subsequently selected for pacemakers instead [3].

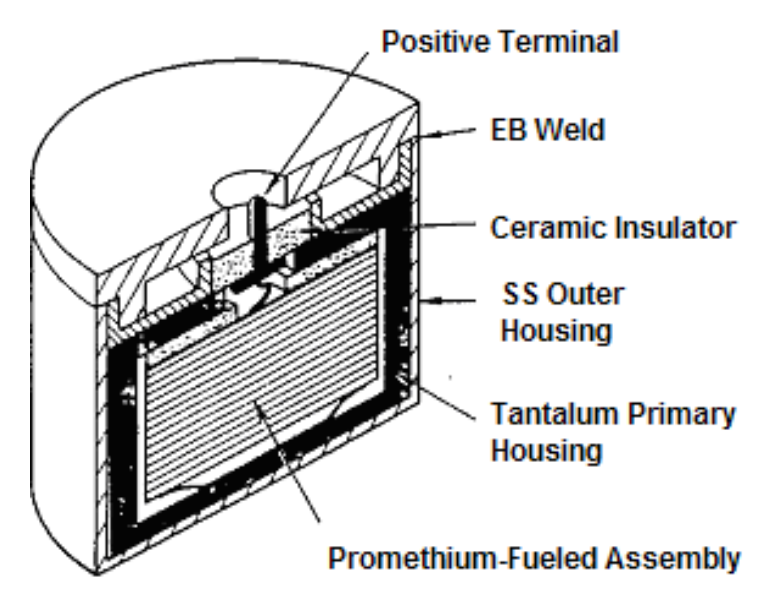

Figure 5. $400 \mu \mathrm{W}$ Betacel battery [3].

Research and development in the last 12 years has concentrated on designs using tritium and $\mathrm{Ni}^{63}$ as beta source and with amorphous silicon (a-Si) and $\mathrm{SiC}$ p-n junctions. In one novel design, the tritium was absorbed into the amorphous silicon p-n junction to passivate silicon dangling bonds. However, as the tritium decayed to helium-3, dangling bonds were created and the conversion efficiency decreased by a factor of two in 18 days [11]. In 2006, Qynergy developed a krypton$85\left({ }^{85} \mathrm{Kr}\right)$ betavoltaic Qyncell ${ }^{\mathrm{TM}}$. The energy density was high, but degraded due to radiation damage and large amounts of defects in silicon carbide [12]. BetaBatt developed a threedimensional porous silicon diode tritium design ( $\mathrm{P}-\mathrm{Si}$ ) but could not achieve a high enough current density [13]. Widetronix has developed nickel-63 $\left({ }^{63} \mathrm{Ni}\right)$ betavoltaic cells with up to $6 \%$ power conversion efficiency [14]. Thicker packaging was required to shield high energy gamma rays from nickel isotopes that could not be removed as shown in Figure 6. In 2008, City Labs successfully produced tritium betavoltaic prototypes, shown in Figure 7, using proprietary material that are currently in operation; City Labs' NanoTritium ${ }^{\mathrm{TM}}$ battery has subsequently been granted the Industry's first and only General License that satisfies all nuclear regulatory guidelines [4]. 


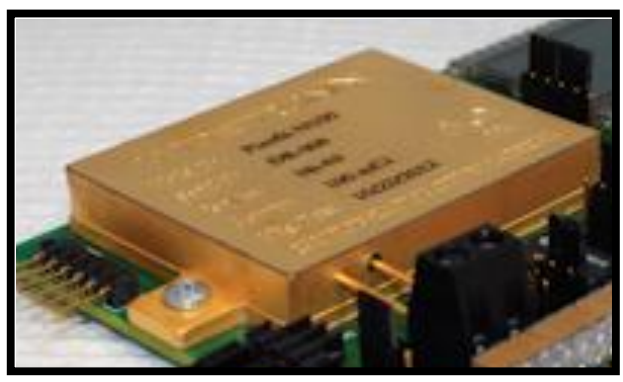

Figure 6. Widetronix Firefly-N betavoltaic.

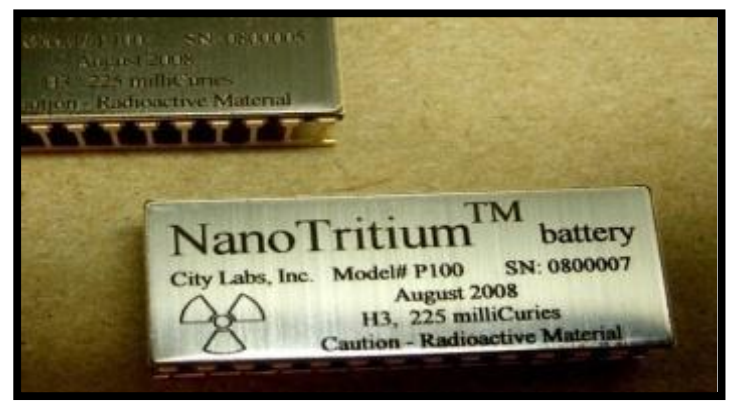

Figure 7. City Labs NanoTritium ${ }^{\mathrm{TM}}$ betavoltaics

\section{Need for a multidiscipline curriculum}

A huge gap exists in the education and research in betavoltaic technology. A multi-disciplinary curriculum to ensure students researching in this technology obtains the necessary knowledge, skills and. experience. Because a betavoltaic consists of a radioactive source and a semiconductor p-n junction, education is needed in material science, mechanical engineering, and electrical engineering as well as in nuclear engineering along with regulations, policies, and perceptions. An understanding of different phenomena to research and develop the critical components is not offered within one school. Thus, a curriculum needs to be adopted to cover the various technical challenges inherent within the following areas.

\subsection{Beta energy spectrum}

Predicting power generation is difficult since beta decay does not occur at specific discrete energies; rather, the emitted electrons form a continuous energy spectrum, which is different for each isotope. Figure 8 shows a generic beta energy spectrum. The average energy is typically one-third the maximum energy. Factors such as self-shielding and low enrichment reduce the quantity of betas that finally reach the betavoltaic converter device. 


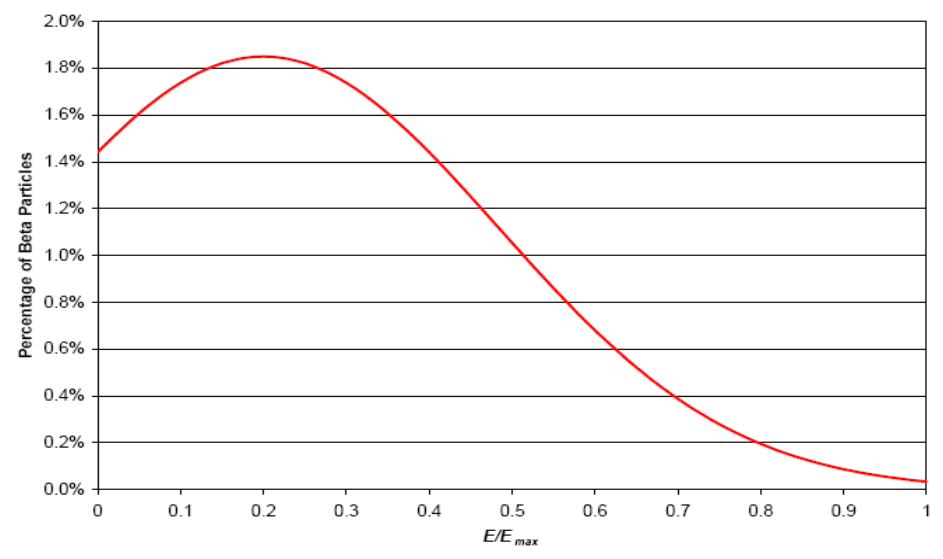

Figure 8. Normalized Beta Energy Spectrum

\subsection{P-N junction}

In choosing the base material for the p-n junction, the material density, band gap energy, and resistivity must be considered. Table 1 shows these characteristics for common semiconductors. High bandgap materials exhibit high open-circuit voltages and low reverse (dark) currents. However, the higher resistivity values indicate a high defect density, which reduces the conversion efficiency. The surface and bulk resistivity of a semiconductor are a function of the number of defects (traps) and impurities [16]. A layer of intrinsic or undoped semiconductor substrate can be left in-between the p-side and $\mathrm{n}$-side to widen the depletion layer.

Table 1. Characteristics of common semiconductor materials at room temperature [17].

$\begin{array}{ccccccc}\text { Property } & \mathbf{S i} & \mathbf{G a A s} & \mathbf{4 H ~ S i C} & \mathbf{G a N} & \text { AlN } & \text { Diamond } \\ \text { Density }\left(\mathrm{g} / \mathrm{cm}^{3}\right) & 2.33 & 5.40 & 3.21 & 6.10 & 3.26 & 3.52 \\ \text { Bandgap }(\mathrm{eV}) & 1.1 & 1.43 & 3.26 & 3.45 & 6.2 & 5.45 \\ \text { Resistivity }(\Omega-\mathrm{cm}) & 1000 & <10^{8} & >10^{12} & >10^{10} & >10^{13} & >10^{13}\end{array}$

\subsection{Beta emitting source}

When selecting a beta emitting isotope, the maximum beta energy and decay half-life are the most important criteria. The beta energy from ${ }^{90} \mathrm{Sr}$ and ${ }^{85} \mathrm{Kr}$ exceeds the minimum dislocation energy of $300 \mathrm{keV}$ which results in semiconductor damage and bremsstrahlung [4]. The 25-day half-life of phosphorus-33 is too short for a long operating life. Therefore, of the three potential isotopes in Table 2, tritium is the most attractive. Tritium is readily available from Ontario Power in Canada and is least expensive at \$3.50/Ci.

Table 2. Potential radioisotopes.

\begin{tabular}{lllllll} 
Isotope & \multicolumn{2}{c}{$\begin{array}{c}\mathbf{T}_{\mathbf{1} 2}(\mathbf{y r})(\mathbf{k e V}) \\
\mathbf{E}_{\mathbf{A V G}}\end{array}$} & $\begin{array}{l}\mathbf{E}_{\mathbf{m a x}} \\
(\mathbf{k e V})\end{array}$ & $\mathbf{C i} / \mathbf{g}$ & $\mathbf{W} / \mathbf{g}$ & $\mathbf{C o s t} / \mathbf{C i}$ \\
Tritium & 12.33 & 5.7 & 18.5 & 9,664 & 0.33 & $\$ 3.5$ \\
Ni-63 & 100.1 & 17.1 & 67 & 59 & 0.006 & $\$ 4,000$ \\
Pr-147 & 2.6 & 65 & 220 & 600 & 0.22 & $\$ 1,000$
\end{tabular}




\subsection{Tritium loading process}

The tritium loading process is the major challenge for direct conversion betavoltaics; it is expensive, time consuming, and lacks control, which results in inconsistent tritium concentrations and damage to films. The absorbing metal is usually deposited on stainless steel foils or silicon substrates. Because only a maximum pressure of 2 bar can only be achieved in present tritium loading systems, the substrates must be heated to temperatures in excess of $400^{\circ} \mathrm{C}$ to initiate diffusion. The metallic film often becomes damaged due to tensile and compressive stresses develop at the thin-film/substrate interface due to tritium absorption and mismatches in thermal expansion as shown in Figure 9 [21].

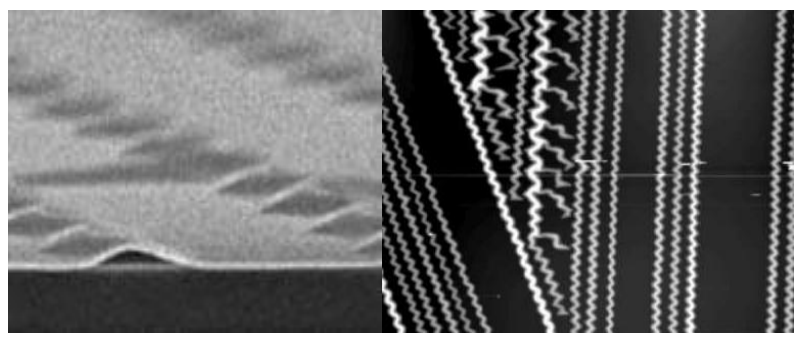

Figure 9. Delamination and buckling of $\mathrm{TiO}_{2}$.

\subsection{Limited Research}

A literature survey was conducted using the Compendex Database accessed through the Purdue University's library portal in June 2013. Variations of betavoltaic were used as the search keyword. The results exhibited some interesting data on when articles were published and who published them as shown in Figure 10 and Figure 11, respectively. Prior to 1976, only 12 articles were published. Since 1976, 138 journal articles were published, and of those, 104 or $75 \%$ were published in the last six years. Over $90 \%$ of the articles were published in the U.S. through 2007. Since then, the number of publications on betavoltaics by U.S. institutions dropped below 20\%, while the number of publications for China increased to over $75 \%$. Canada and Norway have been conducting some research and research is emerging in Korea, Italy, Australia, and Iran.

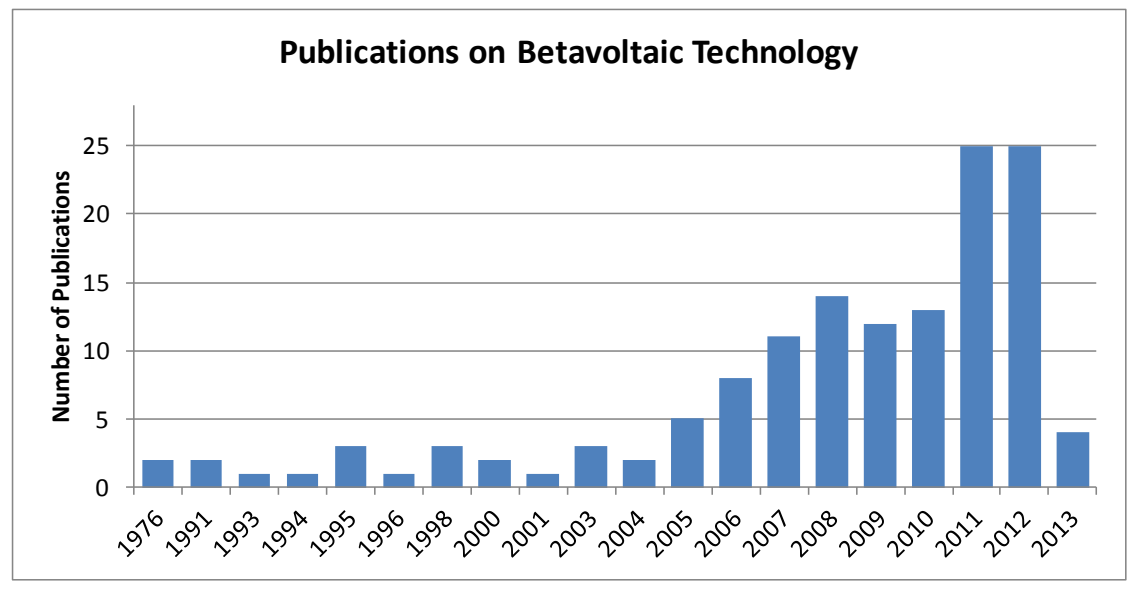

Figure 10. Publications by year since 1976 on Betavoltaic Technology based on Compendex Database generated on June 7, 2013 


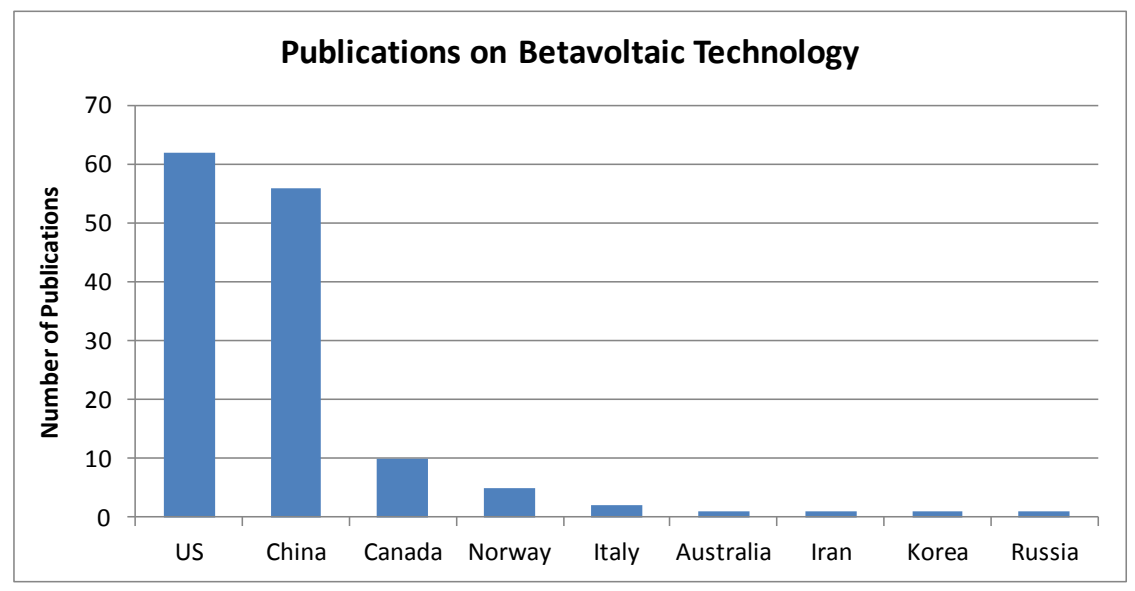

Figure 11. Publications by country since 1976 on Betavoltaic Technology based on Compendex Database generated on June 7, 2013

\section{Integrating research and education}

Because the betavoltaic technology is not mature, work is needed to integrate research with coursework. Most of research conducted in the nuclear engineering department involves thermohydraulics and radiation affects of materials. To increase research and development in betavoltaic cells and other radioisotope power sources, a dedicated laboratory was established at Purdue University to allow students and faculty to conduct research, development, and evaluations on betavoltaic devices. The laboratory currently consists of a hydrogen loading system, energy conversion evaluation system, thermal chamber, fuel cell development and characterization, nuclear pool reactor, and a set of tools to perform modeling and simulation, such as COMSOL, MC-SET and MCNPX. The facility is located in the basement of the electrical engineering building where the Purdue Nuclear reactor is located and is authorized for radioactive materials. Deposition and material analysis instruments are located in the main nuclear engineering facility.

\subsection{Hydrogen Loading system}

A system was needed that could accurately monitor the hydrogen absorption process into metallic films, such as temperature, pressure and resistivity. For a starting point, the system at Greenway Energy located in the Hydrogen Research laboratory at SRNL was used to develop the following preliminary specifications and assumption:

1) Reactor that can hold many samples and withstand high temperatures and pressures of $500^{\circ} \mathrm{C}$ and 1000 psi. Temperature and pressure can be controlled.

2) Small control volume to provide better resolution for pressure measurements.

3) Accurate temperature, pressure, voltage, and current measurements. 
4) Research grade hydrogen (protium) will be used instead of tritium since it behaves the same chemically. However, since the masses are much different, tests can be conducted with deuterium to provide a better basis in predicting the performance of tritium.

The design was an iterative process by building system with available materials and components, evaluation and testing, and replacing with specific components. Accurate volume calculations are necessary, especially at high pressures, in order to have the differential pressure transducer operating within its working range. The schematic of the hydrogen loading system (HLS) is shown in Figure 12. The volume that is measured in experiments is referred as the Control Volume, which consists of volumes 3 and 4. The Reference Volume consists of volume 5 and is used to provide a constant reference pressure on the high side of the differential pressure transducer (DPT). The calibrated $25 \mathrm{cc}$ reservoirs are used to measure the other volumes by successively open valves one at a time. The volume between gas valves, vacuum valve, and control volume is referred as volume 2 and can be used to adjust pressure. Volumes A and $\mathrm{S}$ and small volumes for fine pressure adjustments.

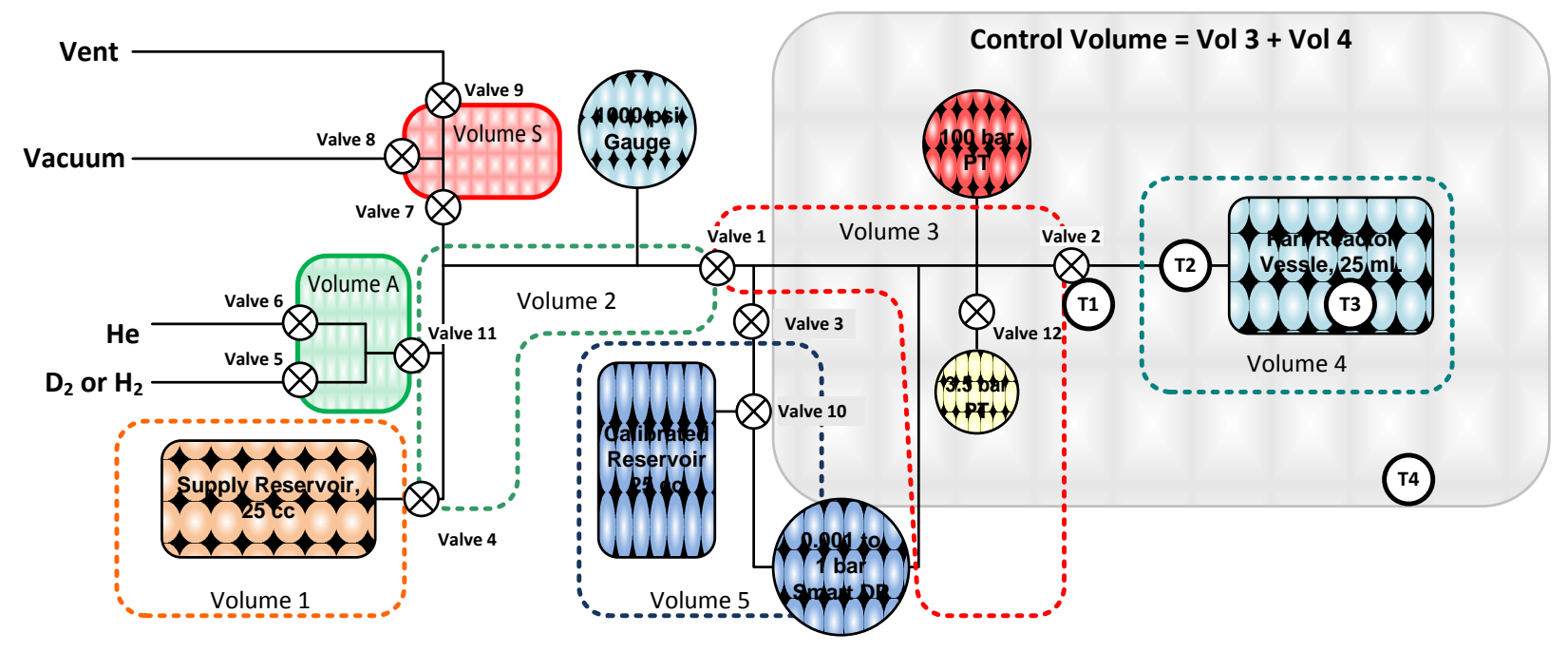

Figure 12. Hydrogen Loading System Schematic

Two thermocouples, $\mathrm{T}_{1}$ and $\mathrm{T}_{2}$, are fastened on the tubing from the Parr reactor to obtain the temperature gradient to calculate precise molar concentrations. Two pressure transducers, 100 bar and 3.5 bar, are used to monitor system pressure while a Honeywell Smart 3000 differential pressure transducer (DPT) with a range of $1 \mathrm{mbar}$ to 1 bar is used to measure the change in pressure due to hydrogen absorption. The amount of hydrogen absorbed is determined by the difference in pressure before and after loading. Pressure and temperature is recorded by a data acquisition system. An example of the loading process is shown in Figure 13, where the system is pressurized with hydrogen first, and then the reactor is heated. The hydrogen activation in the films begins at a temperature of $320^{\circ} \mathrm{C}$. The loading system is currently being validated by a similar system used at Savannah River National Labs. 


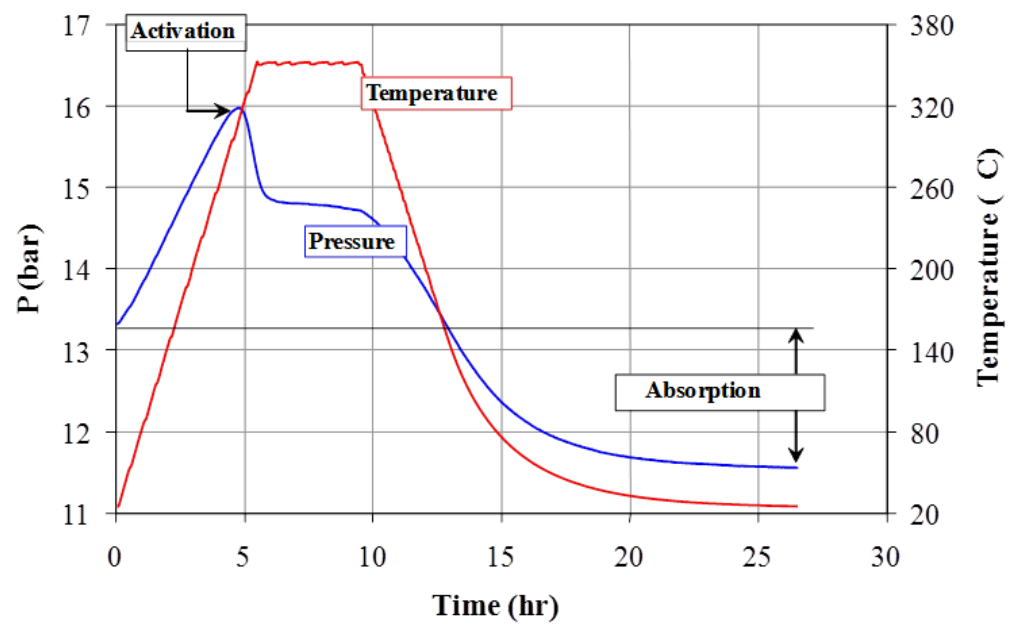

Figure 13. Typical loading profile

Since the samples cannot be visually monitored during the loading process, a four-wire Conax gland was added to the Parr reactor for in situ resistivity $(\rho)$ measurements. Resistivity measurements allow a better understanding of the thermodynamic properties of thin films during tritium loading. The Van de Pauw technique is used to measure the resistivity of the topmost sample using a four-wire probe.

\subsection{Modeling and simulation}

The ability to predict the beta particle flux and its energy distribution from the surface of a metal tritides is of use in advancing betavoltaic technology. A model was developed using a software tool called MC-SET (Monte Carlo - simulation of electrons trajectories in solids) [22], MS Excel, and analytic calculations. MC-SET is used to determine surface flux with its energy distribution in materials that store hydrogen, such as $\mathrm{Mg}, \mathrm{Sc}, \mathrm{Ti}$, and $\mathrm{Pd}$. Mathematical models will use the results to calculate flux and energy distribution by considering isotropic emission, emission energy, exit angle, and material properties. The model has been validated by experimental measurements and reported values.

In Pd films, the beta flux emanating from the surface was predicted. Simulations were run with electrons injected into a semi-infinite Pd slab at $1 \mathrm{keV}$ intervals. The thickness was increased in $50 \mathrm{~nm}$ intervals to where the emission does not change [23]. The simulation results were weighted with the energy distribution probability to produce a beta flux profile versus thickness. In Figure 14, the beta flux does not change beyond a thickness of $500 \mathrm{~nm}$. However, at $100 \mathrm{~nm}$ or one-fifth the thickness, the flux reduces by two times, but only one-fifth of the amount of tritium is used. The maximum thickness where the flux does not change in Sc and Ti is $2000 \mathrm{~nm}$ and $1500 \mathrm{~nm}$ based on material densities. 


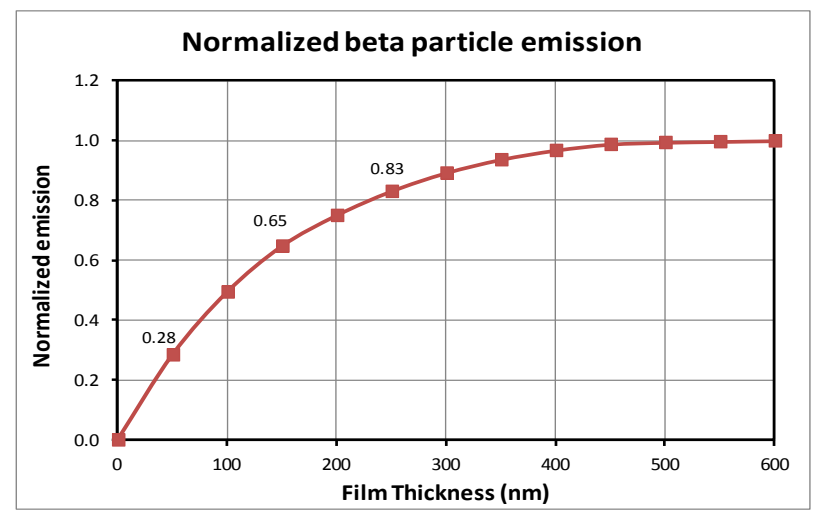

Figure 14. Tritium beta flux in $\mathrm{Pd}$.

\subsection{Low power evaluation system}

Little performance data exists on many emerging energy conversion technologies, such as betavoltaics. Furthermore, affects of temperature, aging, and other phenomena are not known. The system of Figure 15 was designed to evaluate conversion devices and hybrid designs (combination of energy harvesting device and storage device). Betavoltaics and the like are evaluated by their I-V characteristics. The system consists of a temperature chamber, dualchannel source measurement unit, 40-channel digital multimeter, and LabView operating software, and a PC with data acquisition software. Measurements can be made down to nanovolts and femtoamperes. Monitor cables are 24 gauge twisted pair shielded to remove noise. System is backed by UPS for continuous operation in the event of a power failure.

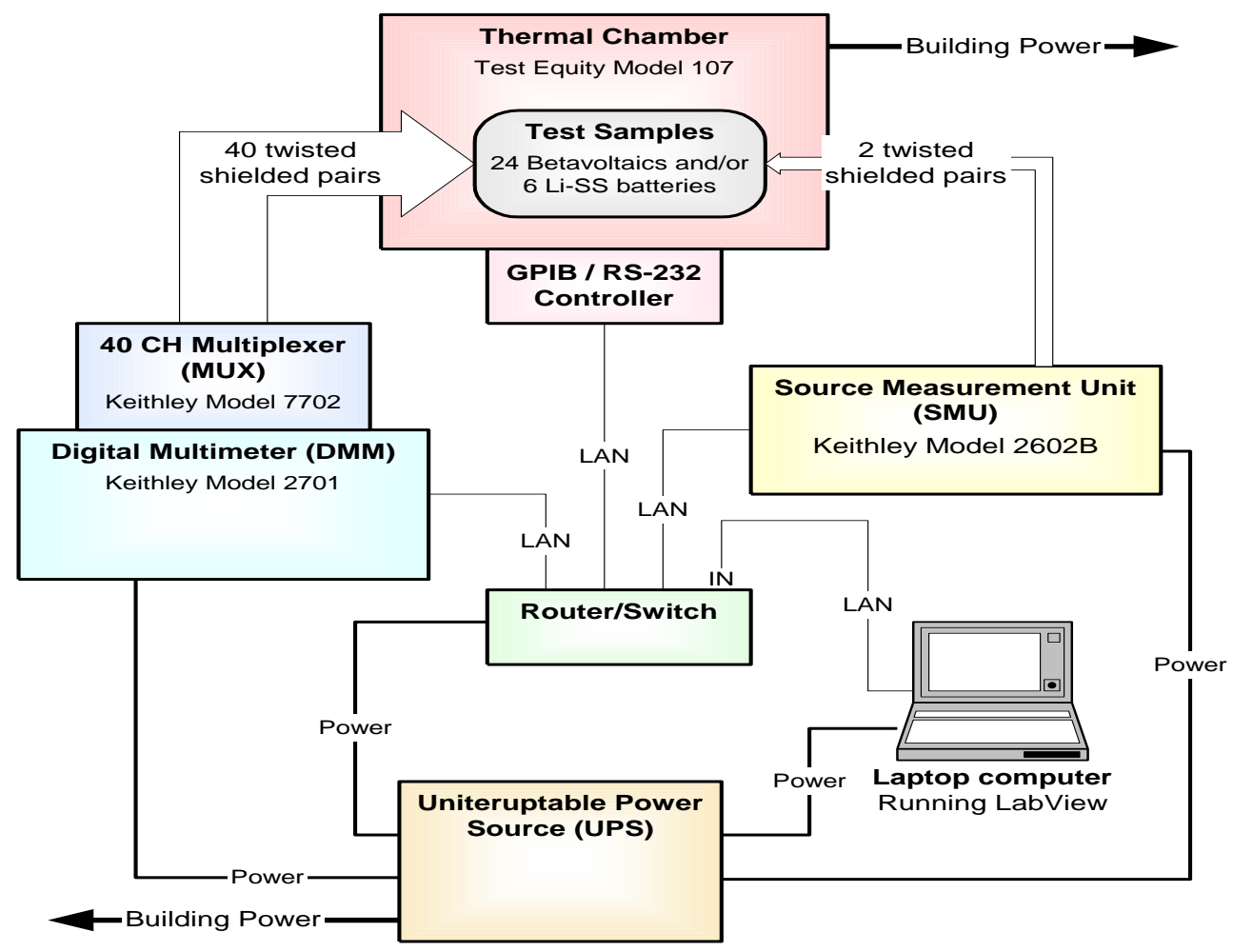

Figure 15. Test System Configuration. 


\section{Approach to relevant research}

Collaboration with Government agencies and industry ensures a high success-rate for advancing the technology, but more importantly, developing a workforce to sustain the technology. Collaboration would identify critical areas of the technology, define applications, and provide funding opportunities to support research and development. Coupled with experimentation, students will have the opportunity to contribute to the advancement of the technology and prepare for successful employment. Tying academia with industry and government in research provides the highest probability of success to further the technology and increase knowledge.

- Prepares students for after graduation while grooming a potential employee for hire.

- Focuses research where funding is available, provides best chance of success.

- Students learn many skills not available in curriculum such as programmatic in writing proposals, cost estimates, planning and scheduling.

- Often much time in graduate school is wasted trying to figure out a project and executing setup of an experiment. Number one problem leading to delays in graduation.

US Government agencies have identified betavoltaics as a disruptive technology that is needed and should be pursued, as evident in reports and solicitations. In October 2013, the Defense Science Board (DSB) issued its report on Technology and Innovation Enablers in 2030. The DSB technology report specifically addresses the use of radionuclide power to lighten the soldiers load. This same report goes into considerable detail to explain other specific use cases tailored for DoD applications in theater. Both initiatives concentrate attention and focus on future disruptive technologies aimed at significantly improving technical superiority for the US warfighter. The research and development of radionuclide power technologies is advancing rapidly. Defense science board recommended investments of $\$ 25 \mathrm{M}$ per year through DARPA and ONR over the next five years to continue the development of this technology as it is still has room for improvements. The Advanced Research Projects Agency-Energy (ARPA-E) is solicitating proposals for nuclear to electrical conversion in the form of betavoltaics. To encourage innovative development of conversion of energy, a program called Innovative Development in Energy-Related Applied Science (IDEAS) was developed. Electrical power sources are increasingly critical enablers of capabilities on the modern battlefield and growing in the future for extreme duration power sources for unattended surveillance sensors and portable power for special operations missions, and enhanced energy density for spacecraft and unmanned vehicles. Betavoltaic technology also has opportunities emerging in detection of corona mass ejection events to protect satellites and space applications, and Betavoltaic sensors would improve the Navy's proliferation monitoring management program in monitoring special nuclear materials (SNMs).

In a recent effort, 24 betavoltaic devices (P100a NanoTritium ${ }^{\mathrm{TM}}$ ) from City Labs and a complement of six Li-SS rechargeable evaluation kits from Cymbet Corporation (Cymbet) are being evaluated. The intent behind this evaluation is two-fold; initially, the study intends to produce an independent performance $\&$ characterization evaluation of each type of power device. Thereafter, merge both power technologies into a viable hybrid power system in order to 
explore the opportunity of developing an exceedingly long-term, temperature insensitive power system as a solution for diminutive low-power applications. The objectives of this effort are 1) to research and develop a state-of-the-art hybrid battery by combining a next generation betavoltaic with a rechargeable battery and capacitor to provide latent and burst power, 2) provide temperature operating limits for betavoltaics and betavoltaic hybrid batteries before they become damaged (expose betavoltaics beyond approved limits), 3) evaluate long-life operation with exposure to established environmental requirements to determine if aging mechanisms beyond radioactive decay exist, and 4) evaluate effects of external forces of radiation (beta, gamma, neutrons, neutrinos and antineutrinos), magnetic flux and electrostatic interference. The objectives are intended to benchmark betavoltaics currently produced by private industry so that betavoltaics can be transitioned and inserted into applications.

The development of this technology, as with any new technology (i.e. lithium batteries) has been slow until now. Interest in the technology is gaining and applications (use cases) are materializing in many sectors within DoD. Although the technology is emerging, it is still not without risk. Current efforts to developmentally mature and advance its TRL (if successful) could offer an enormous payback. More time is needed to properly evaluate this technology to prepare for and transition into applications. Only two companies have invested in the development of this technology and are presently in the position to transition this technology into applications. They cannot do this on their own since many of the applications with the fastest path to transition are out of reach; a collaboration between a DoD lab, industry and academia, such as NSWC Crane, City Labs, and Purdue University is needed to standup the technology and help promote its viability. If applications are not found soon, the technology will fade away and not be available. On a final note, development of this technology has increased in many of the US adversaries in the last seven years, i.e. China, Iran, and North Korea, while development invested by the US has decreased. This technology is obviously important and needs to be implemented immediately.

\section{Acknowledgments}

I would like to thank Naval Surface Warfare Center, Crane Division for the Ph.D. fellowship and support in this research. This work was done while at Purdue University under the direction of my advisor, with the support of my colleagues from Purdue, City Labs, Inc. and Greenway Energy.

\section{References}

1. Adams, T.E., A study of palladium thin-films for radioisotope storage in betavoltaic power sources designs, 2011, Purdue University.

2. Manasse, F.K., J.J. Pinajian, and A.N. Tse, Schottky barrier betavoltaic battery. IEEE Trans. Nucl. Sci, 1976. 23(1).

3. Olsen, L.C. Review of betavoltaic energy conversion. in 12th Space Photovoltaic Research and Technology Conference (SPRAT 12). 1993. NASA Lewis Research Center.

4. Olsen, L.C., P. Cabauy, and B.J. Elkind, Betavoltaic power sources. Physics Today, 2012. 65(12): p. 35-38. 
5. Sun, W., et al., A Three- Dimensional Porous Silicon p-n Diode for Betavoltaics and Photovoltaics. Advanced Materials, 2005. 17(10): p. 1230-1233.

6. Rappaport, P., The electron-voltaic effect in pn junctions induced by beta-particle bombardment. Physical Review, 1954. 93(1): p. 246.

7. Flicker, H., J.J. Loferski, and T.S. Elleman, Construction of a promethium-147 atomic battery. IEEE Transactions on Electron Devices, 1964. 11(1): p. 2-8.

8. Kierstead, H.A., A theory of hydrogen absorption with interactions. Journal of the Less Common Metals, 1984. 96: p. 141-152.

9. Lewis, F.A., Hydrogen in palladium and palladium alloys. International Journal of Hydrogen Energy, 1996. 21(6): p. 461-464.

10. Franco, R. and M.L. Smith. Benefits and Risks of Promethium Battery-Powered Pacemakers. in Advances in Pacemaker Technology. 1974. Erlagen Springer-Verlag.

11. Kosteski, T., et al., Tritiated amorphous silicon betavoltaic devices. IEE Proceedings-Circuits, Devices and Systems, 2003. 150(4): p. 274-81.

12. Eiting, C.J., et al., Demonstration of a radiation resistant, high efficiency SiC betavoltaic. Applied Physics Letters, 2006. 88: p. 064101.

13. Chandrashekhar, M.V.S., et al., Demonstration of a 4 H SiC betavoltaic cell. Applied Physics Letters, 2006. 88: p. 033506.

14. Adams, T., et al., Status of Betavoltaic Power Sources for Nano and Micro Power Applications, in Proc. 45th Power Sources Conference, 11-14 June 2012.2012: Las Vegas, NV.

15. Lamarsh, J.R. and A.J. Barratta, Introduction to nuclear engineering, 2001, Prentice Hall: Upper Saddle River, New Jersey.

16. Neamen, D.A., Semiconductor physics and devices: basic principles2003: Irwin, Inc.

17. Yoder, M.N., Wide bandgap semiconductor materials and devices. IEEE Transactions on Electron Devices, 1996. 43(10): p. 1633-1636.

18. Bower, K.E., Polymers, phosphors, and voltaics for radioisotope microbatteries2002: CRC.

19. Flanagan, T. and W. Oates, The palladium-hydrogen system. Annual Review of Materials Science, 1991. 21(1): p. 269-304.

20. Schroeder, S.L.M. and M. Gottfried, Temperature-Programmed Desorption (TPD) Thermal Desorption Spectroscopy (TDS). 2002.

21. Zhao, F., et al., Buckle delamination of textured $\mathrm{TiO}_{2}$ thin films on mica. Thin solid films, 2005. 489(1): p. 221228.

22. Napchan, E., MC-SET - Monte Carlo Simulation of Electron Trajectories, 2008, [Computer Software].

23. Adams, T.E., A Study of Palladium Thin-Films for Radioisotope Storage in Betavoltaic Power Source Designs, in Nuclear Engineering2011, Purdue University: West Lafayette. p. 91. 